\title{
Excess mortality associated with blindness in leprosy patients in Korea
}

\author{
PAUL COURTRIGHT, $*$ SUNG-HWA KIM, $\dagger$ \\ HO-SUNG LEE, $\&$ \& SUSAN LEWALLEN* \\ *British Columbia Centre for Epidemiologic \& International \\ Ophthalmology, St Paul's Hospital, 1081 Burrard Street, Vancouver, \\ B.C. V6Z 1 Y6 Canada; †Catholic Skin Clinic \& Hospital, Taegu, \\ South Korea; $\ddagger$ Department of Ophthalmology, Catholic University \\ Medical School, Taegu, South Korea
}

\section{Accepted for publication 7 August 1997}

Summary Vision loss and blindness are potential complications of leprosy. There is little data available to indicate the impact of eye complications on life expectancy and quality of life. We sought to determine the relative risk of death in blind leprosy patients compared to nonblind leprosy patients.

A population-based ocular survey of 510 mycobacteriologically negative leprosy patients in rural South Korea, conducted in 1988, formed the study population. After a 7-year period patients were traced to determine their status (alive, dead, lost to follow up).

Blind patients showed a $4 \cdot 8$-fold risk of death, even after adjusting for other factors, compared to nonblind patients. Young blind leprosy patients had the highest relative risk of death. Excess mortality was not associated with any specific cause of blindness, ocular pathology, or type of disease.

Findings from our study suggest that all leprosy patients with ocular disabilities (including those released from antileprosy treatment) should be targeted to receive eye care to prevent vision loss. Particular emphasis should be placed on young patients.

\section{Background}

Evidence was accumulated over 40 years ago demonstrating that leprosy patients, particularly lepromatous patients, have higher age-standardized mortality rates compared to the general, non-leprosy population. ${ }^{1}$ Long-term effects of some of the complications of leprosy, e.g. tropic ulcers, can reduce life span through repeated infection and exposure to economic hardship and malnutrition. Since then, a number of studies have demonstrated 1.5 to 2 fold higher mortality rates among multibacillary patients compared to paucibacillary patients ${ }^{2,3}$ suggesting that leprosy-related reactions and increased frequency of intercurrent diseases, such as tuberculosis predict a higher mortality rate. This may be particularly true where 
medical care is limited. Researchers from the Philippines have also suggested that leprosy patients with an early age at onset of disease (less than 18 years of age) have a higher mortality rate than patients whose onset was when they were older. ${ }^{4}$

Recent evidence in the ophthalmologic literature has suggested that in the general population in developing countries blind people have standardized mortality rates three to four times higher than in the non-blind population. ${ }^{5-7}$ The mechanism by which mortality is increased in blind people has not been adequately explored although it is likely that blindness leads to decreased socioeconomic status, malnutrition, and infections secondary to injury.

Finally, more recently, three population-based surveys have shown that people with cataract (controlling for vision loss) have standardized mortality rates 1.5 to 2 times higher than patients without cataract. ${ }^{8-10}$ Changes in the lens protein are hypothesized to reflect similar system changes that lead to more rapid aging and early mortality.

There is no published information on the relative contribution of disease type, age at leprosy onset, blindness or cataract to mortality in leprosy patients. Thus, our study goal was to determine if the age standardized mortality rate among blind leprosy patients was significantly higher than among nonblind leprosy patients. We also sought to assess the contribution of gender, cataract, type of leprosy, and age at onset of leprosy to increased mortality.

\section{Methods}

In 1988, 593 leprosy patients, resident in 7 resettlement villages in South Korea were encouraged to receive an eye examination. Our study methods have been described previously. ${ }^{11}$ Briefly, in each village best corrected vision was measured followed by a slit lamp examination. A standardized examination form was used to record information on cause of vision loss or blindness and the presence of lid, conjunctival, corneal, uveal, or lens abnormalities. Although all of these patients were mycobacteriologically negative it has been a common practice in Korea to maintain antileprosy therapy and up-to-date records are kept on all patients. Five hundred and ten patients underwent an ophthalmologic examination.

In October 1995, all patient records were reviewed to determine current status (alive and living in the village, died, or moved from the village), regardless of whether they received an eye exam in 1988 or not. Patients no longer living in the village were traced by phone to their new residence. Death certificates are not routinely kept in the leprosy record files and were not available for review.

Age was stratified into three groups and age-adjusted relative risk and confidence limits were calculated. Age at onset of leprosy was divided into those with an onset age of $<18$ and those 18 and over. Stepwise logistic regression modelling was used to determine the risk of death while controlling for the possible confounding effects of other variables. Survival curves based on Kaplan-Meier cumulative probabilities were also calculated; these provide a graphic illustration of the mortality experiences of the two groups.

\section{Results}

At the 7-year follow-up $49(9 \cdot 6 \%)$ of the 510 patients had died. We were unable to locate nine patients. Baseline information on this population in 1988 is given in Table 1. Although only 
Table 1. Study population $(n=510)$ in 1988

Median age
Gender
Median age at onset of leprosy
Median duration of leprosy
Disease type

Clinical information

Best corrected vision

Blind $(<3 / 60)$

Visually disabled $(6 / 18-3 / 60)$

Not visually disabled $(>6 / 18)$

Ocular conditions

Lagophthalmos

Corneal disease

Corneal disease and/or decreased corneal sensation

Chronic uveitis

Leprosy-related eye disease $\dagger$

Cataract or aphakia

Any eye disease $\ddagger$

\author{
53 years \\ $49.0 \%$ male $\quad 51.0 \%$ female \\ 19 years \\ 34 years \\ $77.4 \%$ multibacillary \\ $22.6 \%$ paucibacillary
}

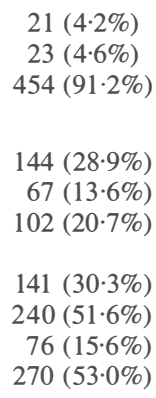

\footnotetext{
* The numbers do not always total to 510 ; clinical information could not be recorded for patients with phthisis bulbi. Also excluded were cases where corneal disease did not permit examination of the iris and lens.

$\dagger$ Leprosy related eye disease is defined as presence of lagophthalmos, lagophthalmos-related corneal disease, decreased corneal sensation, or chronic uveitis.

$\ddagger$ Any eye disease is defined as leprosy-related eye disease or cataract.
}

$4 \cdot 2 \%$ of the study population were blind ( $<3 / 60$ in the better eye), $52 \%$ of those examined had leprosy-related potentially blinding ocular pathology.

Blind patients had an unadjusted risk of death 4.53 times higher $(95 \%$ CI $2 \cdot 72-7 \cdot 54)$ than nonblind patients. The relative risk of death in multibacillary and paucibacillary patients was the same. As expected, increasing age and gender (= males) were both associated with higher mortality. The risk of death of patients with a young age at onset of leprosy was 1.29 times $(95 \%$ CI $0.91,1.81)$ the risk of death of patients with an older age at onset.

Stepwise logistic regression analysis revealed that only age (odds ratio $=1 \cdot 10,95 \% \mathrm{CI}$ $1 \cdot 08,1 \cdot 12, p<0 \cdot 001$ ) and blindness (odds ratio $=4 \cdot 78,95 \% \mathrm{CI} 2 \cdot 87,7 \cdot 96, p=0 \cdot 002$ ) were independently associated with mortality. The graphical association between blindness and mortality, controlling for age, is shown in Figure 1.

Age-stratified analysis revealed that young blind leprosy patients had a mortality ratio $5 \cdot 7$ times higher than among the nonblind cohort (Table 2). There were only 35 patients under 40 years of age, none of whom were blind. Of those patients under 50 years of age $(n=180)$ only one patient was blind in 1988; he died 6 years after the baseline examination.

Excess mortality was not associated with cataract, lagophthalmos, chronic uveitis or any of the other specific ocular pathology findings. The risk of death of patients with visual impairment $(6 / 24-3 / 60)$ was not different than those patients with adequate vision $(\geq 6 / 18)$. 


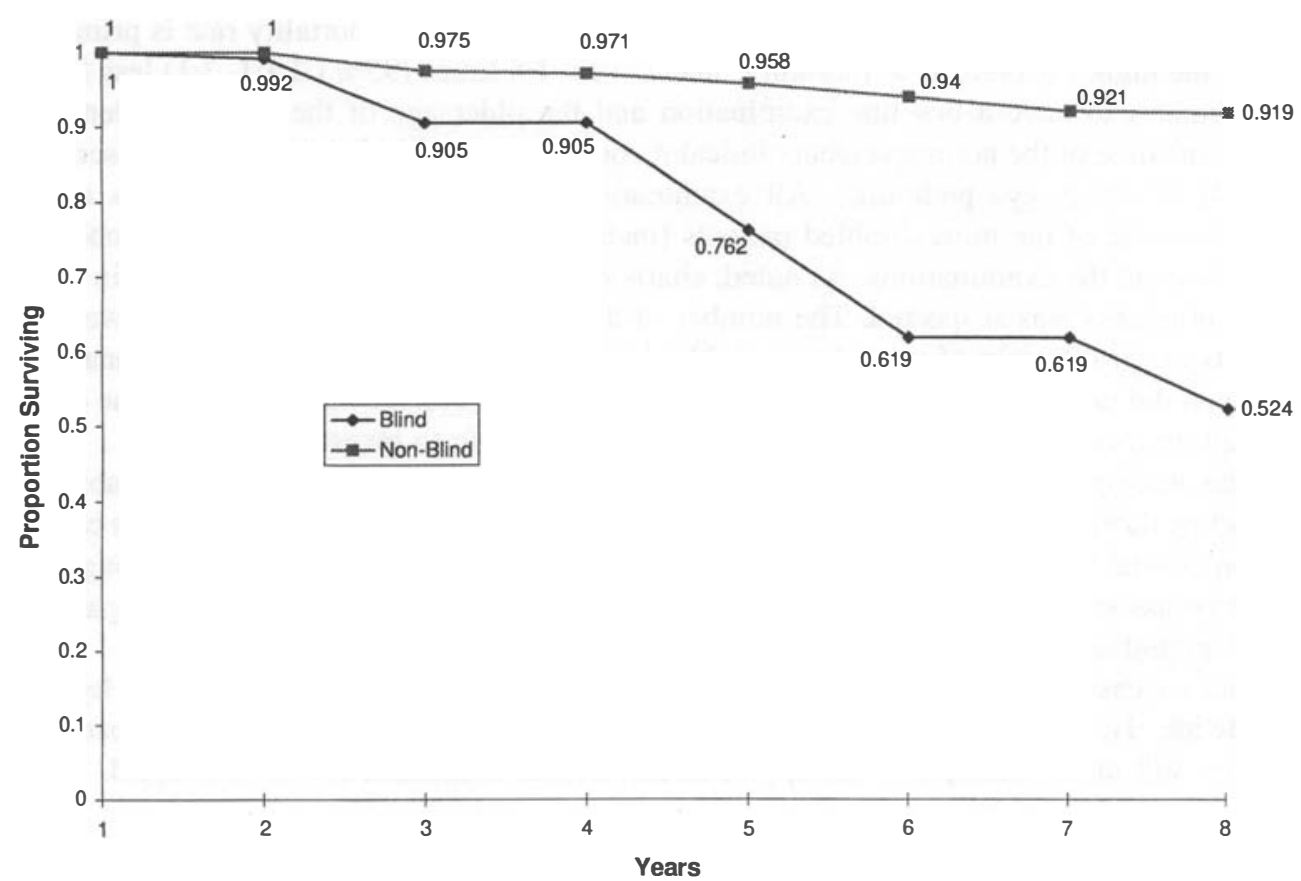

Figure 1. Cumulative survival of blind $(<3 / 60$ in better eye) and nonblind leprosy patients in Korea.

\section{Discussion}

Our study has shown that leprosy patients who are blind have a significantly higher age adjusted risk of death compared to normally-sighted patients. Young blind people have the highest mortality rates. This study has several limitations. Among the 1988 baseline study population, nonrespondents were more likely to be male $(17 \cdot 0 \%)$ than female $(10 \cdot 1 \%)$ $(p=0 \cdot 01)$. They were also slightly older (mean age $=54$ years) compared to respondents (51 years). Mortality among respondents was 97-8/1000, significantly lower than the

Table 2. Age-stratified risk of death among the blind and non-blind

\begin{tabular}{lrrrc}
\hline Age group & No. & Died & Death rate & $\begin{array}{c}\text { Relative risk of death } \\
(95 \% \text { Confidence interval })\end{array}$ \\
\hline$<60$ years & & & & $5 \cdot 6(1 \cdot 6,19 \cdot 5)$ \\
$\begin{array}{l}\text { Blind } \\
\text { Not blind }\end{array}$ & 350 & 18 & $28 \cdot 7$ & \\
$60-69$ years & & & $5 \cdot 1$ & $3 \cdot 3(1 \cdot 4,8 \cdot 1)$ \\
Blind & 9 & 4 & $44 \cdot 4$ & \\
Not blind & 97 & 13 & $13 \cdot 4$ & $1 \cdot 9(0 \cdot 8,4 \cdot 6)$ \\
$70+$ years & & & & \\
Blind & 5 & 3 & $60 \cdot 0$ & \\
Not blind & 28 & 9 & $32 \cdot 1$ & \\
\hline
\end{tabular}


$192 \cdot 3 / 1000$ mortality rate among the nonrespondents. The higher mortality rate is primarily due to the higher overall male mortality; males were 1.8 times $(95 \%$ CI $1 \cdot 1,3 \cdot 1)$ less likely than females to have a baseline examination and the older age of the nonrespondents. A review of some of the nonrespondent clinical records in 1995 revealed that some had serious, possibly blinding, eye pathology. All examinations were done at centralized sites in the villages; some of the most disabled patients (including those with vision related problems) did not attend the examinations. As noted, charts of nonrespondents were reviewed; in some cases, blindness was suspected. The number of blind were relatively small. There were no patients under 40 years of age who were blind. Excluding these patients from the analysis, however, did not significantly change our findings. We do not have information on the cause of death in these patients and this would be a valuable topic to investigate.

The findings from this study suggest that all leprosy patients with ocular disabilities (including those released from antileprosy treatment) should be targeted to receive eye care to prevent vision loss. Particular emphasis should be placed on young patients. Although we found no association between specific ocular diseases and mortality it is likely that patients with lagophthalmos, chronic uveitis, and cataract have a reduced quality of life.

The success of antileprosy therapy has led to a reduction in the incidence of leprosy worldwide. However, it is important to ensure that the visual needs of leprosy patients, whether still under antileprosy therapy or released from treatment, are not neglected.

\section{Acknowledgments}

The 1988 survey was supported by the American Leprosy Missions and German Leprosy Relief Association. The 1995 review was made possible through support of the Robert Cochrane Fund for Leprosy. The authors would also like to thank the staff of the Catholic Skin Clinic \& Hospital for their assistance in carrying out this study.

\section{References}

${ }^{1}$ Guinto RS, Doull JA, de Guia L, Rodriguez JN. Mortality of persons with leprosy prior to sulfone therapy, Cordova and Talisay, Cebu, Philippines. Int J Lepr, 1954; 22: 273-284.

2 Noordeen SK. Mortality in leprosy. Indian Journal of Medical Research 1972; 60: 439-445.

${ }^{3}$ Oleinick A. Survival among leprosy patients with special consideration of cancer as a cause of death. Int J Lepr, 1968; 36: 318-327.

${ }^{4}$ Smith DG and Guinto RS. The association between age on onset and mortality in lepromatous leprosy. Int J Lepr, 1978; 46: 25-29.

5 Kirkwood B, Smith P, Marshall T, Prost A. Relationships between mortality, visual acuity and microfilarial load in the area of the Onchocerciasis Control Programme. Trans R Soc Trop Med Hyg, 1983; 77: 862-868.

6 Prost A, Vaugelade J. La surmortalite des aveugles en zone de savane ouest-af ricaine. Bulletin de l'Organisation mondiale de la Sante, 1981; 59: 773-776.

7 Taylor HR, Katala S, Munoz B, Turner V. Increase in mortality associated with blindness in rural Africa. Bull WHO, 1991; 69: 335-338.

8 Minassian DC, Mehra V, Johnson G J. Mortality and cataract: findings from a population-based longitudinal study. Bull WHO, 1992; 70: 219-223.

9 Street DA, Javitt JC. National five-year mortality after inpatient cataract extraction. Am J Ophthalmol, 1992; 113: 263-268.

10 Thompson JR, Sparrow JM, Gibson JM, Rosenthal AR. Cataract and survival in an elderly nondiabetic population. Arch Ophthalmol, 1993; 111: 675-679.

11 Courtright P, Lewallen S, Lee HS. Comparison of the old and new WHO leprosy disability grading scheme for ocular disabilities. Int Ophthalmol 1991; 15: 295-298. 